\title{
Aortic Arch Branching Patterns in Bicuspid and Tricuspid Aortic Valve Patients
}

\author{
Cornelia Sonia Carr ${ }^{1}$, Shady Ashraf Mohammed ${ }^{1}$, Nazar Mohammed ${ }^{2}$, Lama Shuayb ${ }^{3}$, \\ Maryam Eissa Alkuwari ${ }^{4}$, Abdulaziz Mohammed Alkhulaifi ${ }^{1}$ \\ ${ }^{1}$ Department of Cardiothoracic Surgery, Heart Hospital, Doha, Qatar \\ ${ }^{2}$ Department of Cardiology, Heart Hospital, Doha, Qatar \\ ${ }^{3}$ Qatar Cardiovascular Research Centre, Doha, Qatar \\ ${ }^{4}$ Department of Radiology, Heart Hospital, Doha, Qatar
}

Email address:

noahalkh@gmail.com (C. S. Carr), carr@hamad.qa (C. S. Carr)

${ }^{*}$ Corresponding author

\section{To cite this article:}

Cornelia Sonia Carr, Shady Ashraf Mohammed, Nazar Mohammed, Lama Shuayb, Maryam Eissa Alkuwari, Abdulaziz Mohammed Alkhulaifi. Aortic Arch Branching Patterns in Bicuspid and Tricuspid Aortic Valve Patients. International Journal of Cardiovascular and Thoracic Surgery. Vol. 4, No. 4, 2018, pp. 34-38. doi: 10.11648/j.ijcts.20180404.12

Received: August 6, 2018; Accepted: September 10, 2018; Published: October 10, 2018

\begin{abstract}
The aortic arch and its branches form during the third week of embryogenesis. The most common human pattern has the innominate artery, the left common carotid artery and the left subclavian artery all as separate branches. Large imaging studies have shown $70 \%$ of people have a normal branching pattern with $20 \%$ having a common origin of the innominate artery and left common carotid artery, but these studies were performed without reference to the anatomy of the aortic valve (bicuspid versus tricuspid).Bicuspid aortic valve (BAV) is the commonest congenital cardiac malformation and as the arch branching patterns are developmental in origin we decided to see if the frequency of arch variants in BAV and tricuspid aortic valve (TAV) patients differed, as this has not previously been looked at. We examined Computerised Tomographic aortograms and echocardiograms of BAV and TAV patients to assess the aortic arch branching pattern and any possible association with the valve morphology. $28 \mathrm{BAV}$ and $57 \mathrm{TAV}$ patients were assessed. For BAV the branching patterns were: $86 \%$ normal (24/28) and $14 \%$ abnormal (4/28), and for TAV: 70\% normal (40/57) and 30\% abnormal (17/57). Although this is a small study our TAV group demonstrated comparable normal/abnormal arch variants as the published literature, but the BAV group appears to have fewer arch variants. This is the first study in the literature to look at the arch branching variants when consideration of the aortic valve morphology (BAV versus TAV) is taken into account.
\end{abstract}

Keywords: Bicuspid Aortic Valve, Aortic Arch Branches, Aortic Arch Patterns

\section{Introduction}

The aortic arch and its branches form during the third week of embryogenesis, which involves a complex process. Abnormalities of the arch branching pattern arise by persistence of segments of arches that normally disappear or the disappearance of segments of arches that normally remain, or both [1]. The most common human aortic arch branching pattern has the innominate artery, the left common carotid artery and the left subclavian artery all as separate branches (figure 1). The most common variant branching pattern involves the left common carotid artery arising in a common origin with the innominate artery (figure 2), and the next most common the similar left common carotid artery originating from the innominate artery itself (figure 3). A true bovine arch involves a single common brachiocephalic trunk arising from the arch which then splits into the right subclavian artery, a bicarotid trunk and a left subclavian artery, and is actually extremely uncommon in humans [2]. 

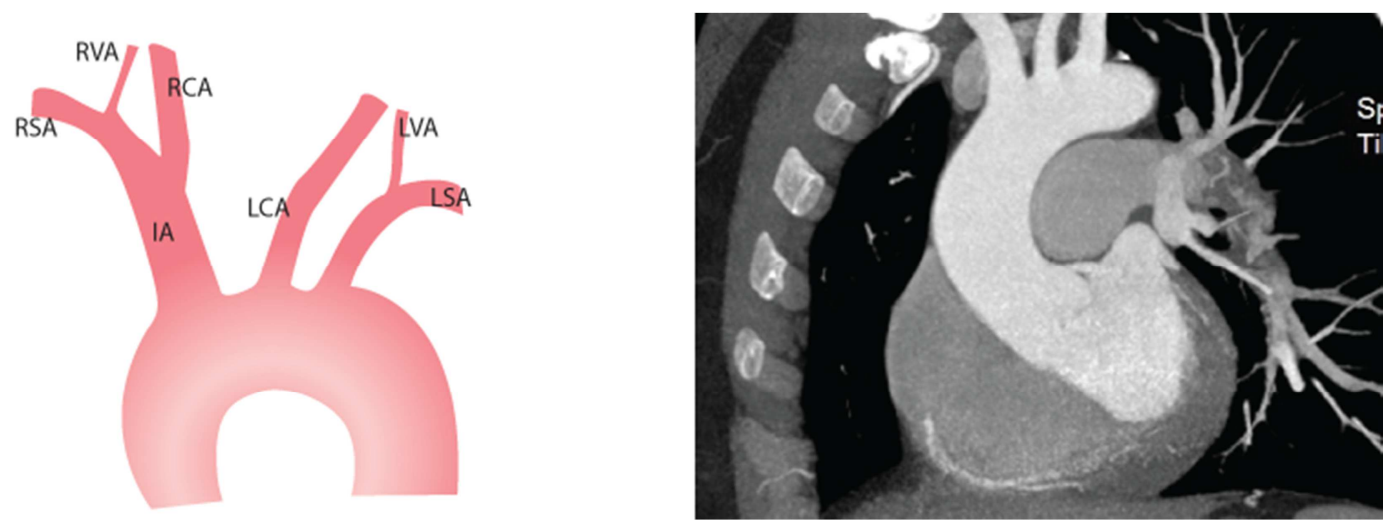

Figure 1. Left common carotid artery and the left subclavian artery all as separate.

Originally the variations of the arch branching patterns were made by post-mortem studies but, more recently large imaging studies have been performed $[4,5]$ confirming that approximately $70 \%$ of people have a normal branching pattern with $20 \%$ having a common origin of the innominate artery and left common carotid artery (as in figure 2) [4, 5], but these studies were performed without reference to the anatomy of the aortic valve (bicuspid versus tricuspid).

Bicuspid aortic valve (BAV) is the commonest congenital cardiac malformation with $1-2 \%$ of the population being affected [6], and is associated with other cardiac anomalies, especially coarctation [6]. BAV is also associated with dilatation of the ascending aorta which is thought to be related to intrinsic pathological properties of the aortic wall and altered flow dynamics through the abnormal valve $[7,8]$, with increased risk of aortic dissection compared to tricuspid aortic valve (TAV) patients $[9,10]$.

As BAV is a common congenital anomaly and the variants of the aortic branching pattern are developmental in origin we decided to see if the frequency of arch variants in BAV and TAV patients differed, as this has not previously been looked at. We examined Computerised Tomographic aortograms (CT) and echocardiograms of BAV and TAV patients to assess the aortic arch branching pattern and any possible association with the valve morphology.

\section{Materials and Methods}

An established BAV patient database was used to retrieve patients. All BAV patients with CT scans of the aorta were examined. During the same period (September 2011-2014) 200 CT aortograms were performed in the Radiology department and from these TAV patients were selected for comparison. Basic demographic data were collected for all the patients. This study was approved by the Institutional Review Board with waiver of consent as all tests had been performed previously. Aortic images, obtained with Multidetector CT aortic angiography (MDCT) using dual source 128 multi slice CT, were further processed by the reporting consultant radiologist for three-dimensional reconstructions to obtain volume rendered images and maximum intensity projections for assessment of the aortic arch branching variation. Echocardiograms of both the BAV and TAV patients were examined to confirm valve anatomy, (images assessed by an echocardiography trained cardiologist, not just reading reports).

\section{Results}

Of the $129 \mathrm{BAV}$ patients in the BAV database 28 had 'readable' (branch pattern could be clearly discerned) CT scans. Fifty-seven (double the number of BAV patients) CT aortograms were selected from the radiology archive (first 57 that were 'readable' from September 2011 that had also undergone echocardiography). The sex and ethnic distributions are shown in tables 1 and 2.

Table 1. Sex and Ethnicity for tricuspid valves.

\begin{tabular}{|c|c|c|c|c|}
\hline Tricuspid & Male & Female & Ethnicity & Numbers \\
\hline \multirow[t]{5}{*}{ Normal $(n=40)$} & 36 & 4 & Africa & 0 \\
\hline & & & Asian Indian Subcontinent & 15 \\
\hline & & & Asian Oriental & 3 \\
\hline & & & Caucasian & 3 \\
\hline & & & MENA Region & 19 \\
\hline \multirow[t]{5}{*}{ Arch Variant $(n=17)$} & 15 & 2 & Africa & 1 \\
\hline & & & Asian Indian Subcontinent & 5 \\
\hline & & & Asian Oriental & 2 \\
\hline & & & Caucasian & 0 \\
\hline & & & MENA Region & 9 \\
\hline
\end{tabular}


Table 2. Sex and Ethnicity for bicuspid valves.

\begin{tabular}{lllll}
\hline Bicuspid & Male & Female & Ethnicity & Numbers \\
\hline Normal $(\mathrm{n}=24)$ & 24 & 0 & Africa & 1 \\
& & & Asian Indian Subcontinent & 7 \\
& & Asian Oriental & 3 \\
& & MENA Region & 13 \\
Arch Variant $(\mathrm{n}=4)$ & 4 & 0 & Africa & Asian Indian Subcontinent \\
& & & Asian Oriental & 2 \\
& & & MENA Region & 2 \\
\hline
\end{tabular}

Eighty-five patients' images were assessed with 28 BAV and 57 TAV. All 85 patients had their echocardiographic images re-examined to verify BAV or TAV morphology. For BAV the aortic branching patterns were: $86 \%$ normal $(24 / 28)$ and $14 \%$ abnormal branching patterns $(4 / 28)$, and for TAV: $70 \%$ normal $(40 / 57)$ and $30 \%$ abnormal branching patterns (17/57). The BAV patients abnormal patterns were all the left

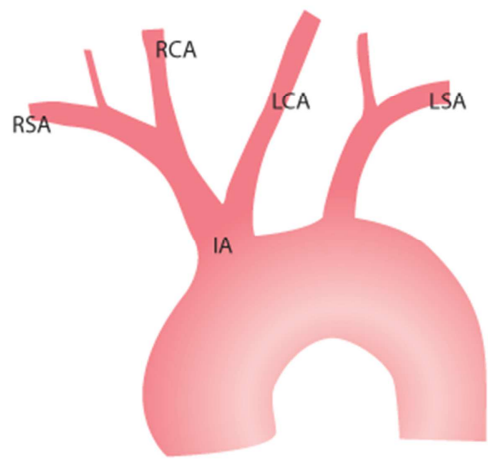

Figure 2. The type left common carotid artery common origin with innominate artery.
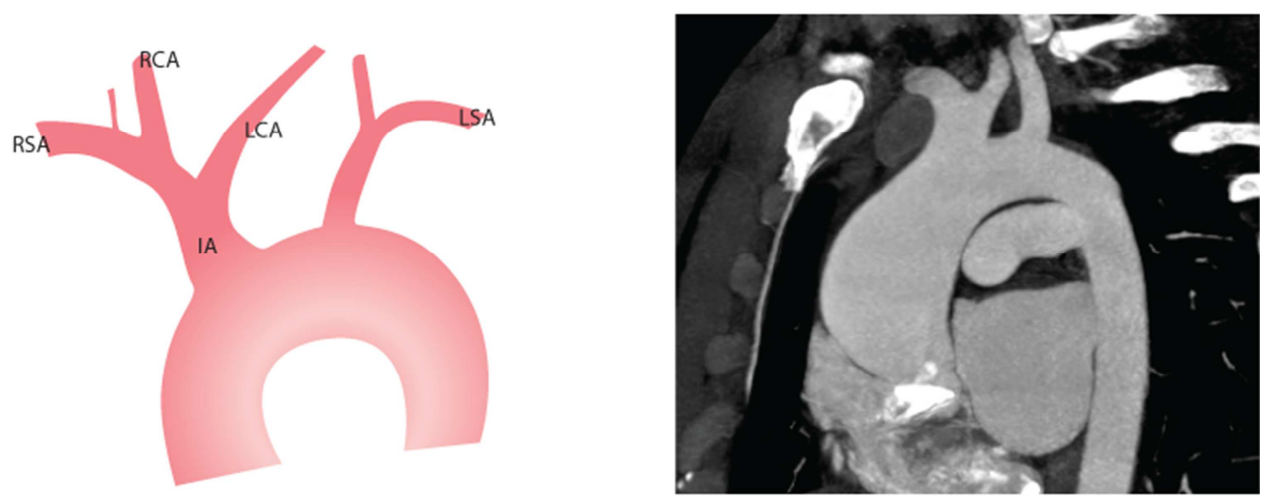

Figure 3. Left common carotid arising from the innominate artery.

Other anomalies found in our group include 1 coarctation (BAV patient) and 1 dissection (BAV patient). The valve morphology was discernable in $42(49 \%)$ of the 85 CT scans.

\section{Discussion}

\subsection{Embryologically}

The six pairs of aortic arches are a series of vessels that connect on each side the aortic sac with the corresponding

common carotid artery common origin with innominate artery (as in figure 2), whereas the TAV patients had 16 of the type left common carotid artery common origin with innominate artery (as in figure 2) and one left common carotid arising from the innominate artery (as in figure 3 ). There were no true bovine variants in our group.

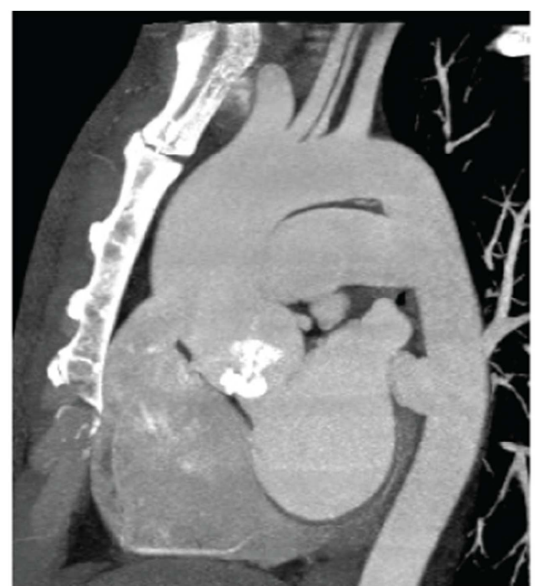


branching pattern of arch of aorta may occur, as observed in present study. The proximal part of the third aortic arch normally gets extended and absorbed into the left horn of aortic sac. If it gets absorbed into the right horn of the aortic sac, that also results in a variable branching pattern $[13,14]$. Direct origin of the left vertebral artery from the upper convex surface of the arch of aorta between the origins of the left common carotid and left subclavian arteries may be explained as increased absorption of embryonic tissue of the left subclavian artery between the origin of the arch of aorta and the vertebral artery. Variations, when there are more than three branches originating from the arch of aorta may include the vertebral arteries $[15,16]$.

\subsection{Gross Anatomy}

The aortic arch represents the continuation of the ascending aorta and is nominally defined as starting at the level of the transthoracic plane of Ludwig, a horizontal plane from the sternomanubrial angle to the T4 vertebral body. The sternomanubrial joint is the same level as the second sternocostal articulation. It courses in a narrow arch from ventral to dorsal and from right to left such that at the end of the arch it sits to the left of midline, adjacent to the thoracic vertebral column. Its peak is at the T3/4 level. Three main branches originate from the upward convexity of the arch in the majority $(75 \%)$ of patients. In order from proximal to distal the branches are: 1- brachiocephalic trunk or artery (innominate artery) which goes on to divide into the right subclavian and right common carotid arteries, the aortic arch can be divided by the brachiocephalic trunk into "proximal" and "distal" portions ${ }^{3}$. 2- left common carotid artery, 3-left subclavian artery. just beyond the last branch, the aortic isthmus represents a minor narrowing at the site of the ligamentum arteriosum, which runs between the undersurface of the aortic arch and the terminal pulmonary trunk, this ligament represents the obliterated foetal ductus arteriosus and due to this attachment, this represents the site of the majority of thoracic aortic injuries when the body undergoes significant deceleration. The arch terminates at the lower border of $\mathrm{T} 4$ where it continues as the descending aorta, in the plane of Ludwig, a horizontal plane from the sternomanubrial angle to the T4 vertebral body [16].

\subsection{Bicuspid Aortic Valve}

Has previously been quoted as having a related aortopathy, but is this embryological or functional (related to intrinsic wall properties) in origin? [7,8]. If in fact BAV patients have fewer arch variations it may support the idea that the aortopathy is related to functional changes in the wall rather than embryological origins (excluding coarctation). In our group, it does not appear to affect the embryological development of the branches of the aortic arch and we actually appear to have fewer arch variants in the BAV group. Although this is a small study our TAV group had similar percentages of normal (70\%) and abnormal (30\%) arch variants as the reported literature $[1,2,4,5]$. Our BAV group is small and we will expand it further in the future but the results at this stage suggest no increase, in fact a possible decrease in arch variants compared to the TAV patients. Despite this it would not be possible to exclude an embryologically based pathological change to the aorta in BAV patients.

\subsection{In a Study on the patterns of Aortic Arch}

In American white and Negro stocks by De Garis CF et al $25 \%$ of blacks were found to have a common origin for innominate artery and left common carotid artery. The author has also reported an incidence of $10 \%$ in blacks where the left common carotid artery arises from the Innominate artery. [3], but these studies would be difficult to repeat due to widespread racial mixing nowadays and could only be looked at in isolated communities with a single racial group. In Qatar $60 \%$ of the population is originally from the Asian Indian Subcontinent so there may in fact be a greater prevalence of Bicuspid valves in the MENA region patients and also Arch Variants appear to be more common in the MENA region patients. The two large radiological studies that have looked at the aortic arch anatomy showed that approximately $70 \%$ of patients had normal configuration of the arch vessels and $20 \%$ had a common origin of the innominate artery and left common carotid artery [7,8], but these studies were performed without reference to the anatomy of the aortic valve. This is the first study in the literature to look at the arch branching variants when consideration of the aortic valve morphology (BAV versus TAV) is taken into account.

\section{Conclusion}

Aortic arch branching pattern variations are usually asymptomatic but are significantly more common in patients with thoracic aortic disease (aneurysm formation, dissection, susceptibility to transection), and may therefore act as a marker for the development of thoracic aortic disease in the future (Dumfarth, Wanamaker) [17, 18]. As BAV patients are known to have more dilatation and dissection the combination of BAV with arch branching variants may warrant closer surveillance than other patients. Thus, aortic arch branch pattern variants are not clinically irrelevant as they may indicate an increased likelihood of the development of thoracic aortic disease in the future. Also with the increasing use of transcatheter procedures replacing surgical interventions the exact anatomy and its impacts will become more important. At the time of aortic arch surgery, it is important to be aware of any anomalies as they can influence cannulation and perfusion strategies as well as the repair performed.

\section{Acknowledgements}

We would like to thank Mr Mohammed Abdulsamad for diagrams. 


\section{References}

[1] Kau T, Sinzig M, Gasser J, Lesnik G, Rabitsch E, Celedin S, Eicher W, Illiasch H, Hausegger KA. Aortic Development and Anomalies. Semin Intervent Radiol 2007;24(2):141-152.

[2] Layton KF, Kallmes DF, Cloft HJ, Lindell EP, Cox VS. Bovine aortic arch variant in humans: clarification of a common misnomer. American Journal of Neuroradiol 2006;27:1541-42.

[3] N. shakuntala Rao, k. Sujatha, K Meera, H.R. Krishna Rao. A comparative study on the structure and functions of aorta in man and ruminant animals. International Journal of Anatomy and Research, Int J Anat Res 2016, Vol 4(4):3194-98.

[4] Jakanani GC, Adair W. Frequency of variations in aortic arch anatomy depicted on multidetector CT. Clinical Radiology 2010;65:481-487.

[5] Shakeri A, Pourisa M, Deldar A, Goldust M. Anatomic variations of aortic arch branches and relationship with diameter of aortic arch by 64-row CT angiography. Pak J Biol Sci 2013;16(10):496-500.

[6] Nistri S, Basso C, Marzari C, Mormino P, Thiene G. Frequency of bicuspid aortic valve in young male conscripts by echocardiogram. Am J Cardiology. 2005;96(5):718-721.

[7] Mahadevia R, Barker AJ, Schnell S, Entezari P, Kansal P, Fedak PW, Malaisrie SC, McCarthy P, Collins J, Carr J, Markl M. Bicuspid aortic cusp fusion morphology alters aortic threedimensional outflow patterns, wall shear stress, and expression or aortopathy. Circ 2014;129(6):673-82.

[8] Phillippi JA, Green BR, Eskay MA, Kotlarczyk MP, Hill MR, Robertson AM, Watkins SC, Vorp DA, Gleason TG. Mechanism of aortic medial matrix remodeling is distinct in patients with bicuspid aortic valveJ Thorac Cardiovasc Surg 2014;147(3):1056-64.

[9] Michelena HI, Khanna AD, Mahoney D, Margaryan E, Topilsky Y, Suri RM, Eidem B, Edwards WD, Sundt TM 3rd,
Enriquez-Sarano M. Incidence of aortic complications in patients with bicuspid aortic valves. JAMA 2011;306(10):1104-12.

[10] Eleid MF, Forde I, Edwards WD, Maleszewski JJ, Suri RM, Schaff HV, Enriquez-Sarano M, Michelena HI. Type A aortic dissection in patients with bicuspid aortic valves: clinical and pathological comparison with tricuspid aortic valves. Heart 2013;99(22):1668-74.

[11] Fazel SS, Mallidi HR, Lee RS, Sheehan MP, Laing D, Fleischman D, Herfkens R, Mitchell RS, Miller DC. The aortopathy of bicuspid aortic valve disease has distinctive patterns and usually involves the transverse aortic arch. The Journal of Thoracic and Cardiovascular Surgery 2008;135:901-7.

[12] Moore KL, Persaud TV. The developing human: clinically oriented embryology. 8th ed. Philadelphia: Saunders Elsevier; 2008. pp. 305-306.pp. 316-325.

[13] Sadler TW. Langman's medical embryology. 10th ed. Philadelphia: Lippincott Williams \& Wilkins; 2006. pp. 173175.pp. 180-185.

[14] Nelson ML, Sparks CD. Unusual aortic arch variation: distal origin of common carotid arteries. Clin Anat. 2001;14:62-65. [PubMed].

[15] Satyapal KS, Singaram S, Partab P, Kalideen JM, Robbs JV. Aortic arch branch variations: case report and arteriographic analysis. S Afr J Surg. 2003;41:48-50.

[16] Dr Sachintha Hapugoda and A.Prof Frank Gaillardet al. Aortic arch. www.radiopaedia.org.

[17] Julia Dumfarth, Alan S. Chou BA, Bulat A. Ziganshin et all. Atypical aortic arch branching variants: A novel marker for thoracic aortic disease. The journal of thoracic and cardiovascular surgery Volume 149, Issue 6. June 2015, Pages 1586-1592.

[18] K.M. Wanamaker, C.C. Amadi, J.S. Mueller, R.J. Moraca. Incidence of aortic arch anomalies in patients with thoracic aortic dissections. J Card Surg, 28 (2013), pp. 151-154. 